\title{
Association of antigen processing machinery and HLA class I defects with clinicopathological outcome in cervical carcinoma
}

\author{
Akash M. Mehta · Ekaterina S. Jordanova • \\ Gemma G. Kenter · Soldano Ferrone • \\ Gert-Jan Fleuren
}

Received: 19 February 2007 / Accepted: 20 June 2007/Published online: 12 July 2007

(C) Springer-Verlag 2007

\begin{abstract}
HLA class I loss is a significant mechanism of immune evasion by cervical carcinoma, interfering with the development of immunotherapies and cancer vaccines. We report the systematic investigation of HLA class I and antigen processing machinery component expression and association with clinical outcome. A tissue microarray containing carcinoma lesions from 109 cervical carcinoma patients was stained for HLA class I heavy chains, $\beta_{2}$-microglobulin, LMP2, LMP7, LMP10, TAP1, TAP2, ERAP1, tapasin, calreticulin, calnexin and ERp57. A novel staining evaluation method was used to ensure optimal accuracy and reliability of expression data, which were correlated with known clinicopathological parameters. Partial HLA class I loss was significantly associated with decreased 5-years overall survival (61\% vs. $83 \%$ for normal expression; $P<0.05)$ and was associated with decreased 5-years disease-free survival (DFS) (65\% vs. $82 \%$ for normal expression; $P=0.05)$. All APM components except LMP10, calnexin and calreticulin were down-regulated in a substantial number of cases and, except ERAP1, correlated
\end{abstract}

\footnotetext{
A. M. Mehta - E. S. Jordanova ( $\square)$.

G.-J. Fleuren

Department of Pathology,

Leiden University Medical Centre,

P1-40, L1-Q, 2333 ZA Leiden, The Netherlands

e-mail: k.jordanova@lumc.nl

G. G. Kenter

Department of Gynaecology,

Leiden University Medical Centre,

Leiden, The Netherlands

S. Ferrone

Department of Immunology,

Roswell Park Cancer Institute,

Buffalo, NY, USA
}

significantly with HLA class I down-regulation. LMP7, TAP1 and ERAP1 loss was significantly associated with decreased overall and (except LMP7) DFS $(P<0.05$ and 0.005 , respectively). ERAP1 down-regulation was an independent predictor for worse overall and DFS in multivariate analysis (HR 3.08; $P<0.05$ and HR 2.84; $P<0.05$, respectively). HLA class I and APM component downregulation occur frequently in cervical carcinoma, while peptide repertoire alterations due to ERAP1 loss are a major contributing factor to tumour progression and mortality.

Keywords Antigen processing machinery - ERAP1 . Cervical carcinoma

\section{Introduction}

Tumour cells utilize several mechanisms to escape immune-mediated recognition and destruction [1]. Loss of surface-expressed human leukocyte antigen (HLA) class I molecules is particularly important, as this enables tumour cells to evade recognition and lysis by cytotoxic T-lymphocytes [2-5]. These molecules consist of a glycoprotein heavy chain (encoded by genes within the HLA regions of chromosome $6 \mathrm{p})$ and a $\beta_{2}$-microglobulin $\left(\beta_{2}-\mathrm{m}\right)$ light chain (encoded on chromosome 15q) [6, 7].

Defects in HLA class I expression are caused by multiple mechanisms, including loss of heterozygosity at chromosome $6 \mathrm{p} ; \beta_{2}-\mathrm{m}$ or HLA class I mutations; and defective expression and/or function of components of the antigen processing machinery (APM) [8-10]. The APM is the combination of cellular processes responsible for the presentation of endogenous peptides by HLA class I molecules. These peptides are generated by specialized immunoproteasomes (composed of LMP2, LMP7 and 
LMP10 subunits); and transported from the cytoplasm to the endoplasmic reticulum by TAP (transporter associated with antigen presentation) which is composed of TAP1 and TAP2 subunits. Transported peptides undergo lengthspecific trimming by ERAP1 (endoplasmic reticulum aminopeptidase associated with antigen presentation 1) and are loaded onto empty HLA class I molecules (with involvement of tapasin and the chaperone molecules calnexin, calreticulin and ERp57) [11-14].

Although various in vitro studies have demonstrated partial or total APM component loss in human malignancies, the extent to which this occurs in vivo is largely unknown. Two recent reports describe down-regulation of several APM components in head and neck squamous cell carcinoma and astrocytoma, and demonstrate an association of these defects with prognosis $[15,16]$. As HLA class I-restricted processing and presentation of endogenous peptides is crucial to the generation of a virus-specific cytotoxic T-lymphocyte-mediated reaction, APM defects are assumed to contribute significantly to virus-mediated carcinogenesis. Several studies have confirmed interference in various APM processes by viral peptides [17]. However, the extent to which defective expression of APM components contributes to HPV-induced cervical carcinogenesis remains to be established. One study has assessed the expression of various APM components, although only at the mRNA level [18]. Other studies of APM component expression in cervical carcinoma were largely limited to TAP1 and TAP2 and encompassed relatively small sample sizes. Moreover, the expression data yielded by these studies are conflicting, with TAP1 loss being reported in $0-50 \%$ of cases [18-20]. Though TAP1 and HLA class I down-regulation has occasionally been shown to be associated with progressive malignant transformation [21-24], a comprehensive study of the association of APM defects with prognostic parameters has yet to be performed.

Since information regarding defective APM component expression in cervical cancer can contribute to the development of $\mathrm{T}$ cell-based immunotherapies, we have investigated the expression of all known APM components in a large number of cervical cancer specimens using a tissue microarray (TMA)-based approach [25, 26]. Additionally, we demonstrate for the first time a negative association of APM defects with prognosis and other clinicopathological variables.

\section{Materials and methods}

Clinical characteristics and tissue samples

From 109 patients with cervical carcinoma who underwent radical hysterectomy with bilateral pelvic lymphadenectomy (by the same surgical team) between 1985 and 1999, formalin-fixed, paraffin-embedded tissue blocks were retrieved from the archives of the Department of Pathology, Leiden University Medical Centre, The Netherlands. Twenty-six adenocarcinomas and 83 squamous carcinomas were obtained. These cases were not consecutive, but chosen on the basis of availability of sufficient material. The use of clinical material was approved by the institutional review board according to the guidelines of the Dutch Federation of Medical Research Associations.

All patients were inhabitants of The Netherlands and had not received pre-operative radiotherapy or chemotherapy. Mean age was 48.5 years, the youngest patient being 24 years and the oldest 87 years at the time of surgery. Follow-up of these patients until 2005 provided information concerning recurrence rate and performance state.

Tissue microarray construction

The archival slides for all the cases were reviewed; a slide containing representative tumour was selected, and an area of tumour was encircled on the slide. The corresponding tissue blocks were recovered from the archives and the selected area on the slide was circled on the block for tissue microarray construction. Using a manual tissue microarrayer (Beecher Instruments, Silver Spring, MD, USA), the area of interest in the donor block was cored with a 0.6-mm diameter needle and transferred to a recipient paraffin block. The microarray was constructed with a threefold redundancy (three spots for each patient) to increase accuracy. The finalized arrays were then cut into $3 \mu \mathrm{m}$-thick sections and mounted on glass slides using an adhesive tape-transfer system (Instrumedics Inc., Hackensack, NJ, USA) with ultraviolet cross-linking.

\section{Antibodies}

The mouse monoclonal antibody (mAb) HCA2 which recognizes a determinant expressed on $\beta_{2}$-m-free HLA-A (excluding HLA-A24), HLA-B7301 and HLA-G heavy chains $[27,28]$, the $\mathrm{mAb} \mathrm{HC} 10$ which recognizes a determinant on all $\beta_{2}$-m-free HLA-B and HLA-C heavy chains and on $\beta_{2}$-m-free HLA-A10, -A28, -A29, -A30, -A31, -A32 and -A33 heavy chains [27, 29], the LMP2-specific mAb SY1 [30], the LMP7-specific mAb HB-2 [30], the LMP10-specific mAb TO-7 [30], the TAP1-specific mAb NOB-1 [31], the TAP2-specific mAb NOB-2 [31], the calnexin-specific mAb TO-5 [32], the calreticulin-specific mAb TO-11 [32], the Erp57-specific mAb TO-2 [32] and the tapasin-specific mAb TO-3 [32] were developed and characterized as described. 
The rabbit polyclonal anti- $\beta_{2}-\mathrm{m}$-antibody A-139 was purchased from DAKO, Copenhagen, Denmark. The rabbit polyclonal anti-ERAP1 antibody has been described previously and was kindly provided by Dr. M. Tsujimoto, RIKEN, Wako, Saitama, Japan [33-35].

Immunohistochemical staining

The slides were dried for $16 \mathrm{~h}$ at $56^{\circ} \mathrm{C}$ before being dewaxed with standard xylene and rehydrated through graded alcohols into water. Standard immunohistochemical staining was performed as described previously [36]. Stromal cells, including infiltrating leukocytes, were used as internal positive controls. Negative control sections of each specimen were processed with omission of the primary antibodies.

\section{Evaluation of TMA immunostaining}

Two researchers (A.M. and E.J.) evaluated all patient cores, and scores were averaged to produce a single evaluation with the scorers being blind to the source of the samples. When slides were scored differently, which occurred rarely $(<5 \%)$, a consensus was reached by simultaneous evaluation. Staining was scored semi-quantitatively by the quality control system proposed by Ruiter et al. [37]. The intensity and percentage of positive cells in the tumour and the stromal cells surrounding the tumour were determined. The intensity of staining was scored as $0,1,2$, or 3 indicating absent, weak, clear, or strong expression, respectively. The percentage of positive cells was scored as 0 for $0 \% ; 1$ for $1-5 \% ; 2$ for $5-25 \%$; 3 for $25-50 \%$; 4 for $50-75 \%$ and 5 for $75-100 \%$. The sum of both scores was used to identify three categories of expression: normal expression (total score 7-8), partial loss (3-6) and total loss (0-2). Immunohistochemical staining demonstrated strong positive expression of all examined markers in stromal tissue and tumour-infiltrating inflammatory cells, thereby providing an internal positive control.

\section{Statistical analysis}

The Chi-square test or, where appropriate, Fisher's exact test was used for evaluation of associations between expression and clinicopathological parameters. All statistical analyses were performed with the SPSS Version 12 software package. Five-year survival rates were calculated and survival rates were compared according to the KaplanMeier method using the log rank test, while multivariate analysis of survival was performed according to Cox proportional hazard models. Overall survival (OS) was defined as survival till death due to cervical carcinoma (the two patients that dyed from other causes were excluded), i.e. disease-specific survival, while disease-free survival (DFS) was defined as time to disease recurrence, metastasis, or disease-specific death. All tests were two-sided and the significance level was set to $5 \%$.

\section{Results}

\section{Clinical features}

Of the total group of 109 patients, 52 were diagnosed as FIGO stage IB1, 30 as IB2, 21 as FIGO IIa and 2 as FIGO IIb. For four patients no FIGO staging was available. Fiftyfive patients $(50 \%)$ received post-operative radiotherapy due to narrow tumour-free margins of excision, lymph node metastases, vaso-invasive growth, parametrial infiltration, depth of infiltration exceeding $15 \mathrm{~mm}$ and/or tumour size exceeding $4 \mathrm{~cm}$. Twenty-seven patients had lymph node metastases. Fifty-one patients (58\%) were HPV16-positive, 21 (24\%) were HPV18-positive, while the remaining patients were positive for HPV31, HPV33, HPV45, HPV52 or HPV68. At the end of follow-up, 80 patients were alive, 2 suffered from recurrent disease, 3 had a metastasis, 22 had died of the disease and 2 had died of causes unrelated to the primary disease. The median follow-up time was 45 months.

\section{HLA class I and APM component expression}

Representative staining patterns for the HLA class I heavy chains and various APM components are shown in Figs. 1 and 2. Expression of the HLA class I heavy chains and $\beta_{2}-\mathrm{m}$ is summarized in Table 1 . Of the 109 cases, eight exhibited total $\beta_{2}-\mathrm{m}$ loss; correspondingly, these cases were also negative for HLA heavy chain cell surface expression. As HLA class I expression, and therefore tumour immunogenicity, is not affected by the APM in a $\beta_{2}$-m-negative background these eight cases were excluded from further analysis of APM effects. Furthermore, as HLA class I expression is regulated by the APM in a $\beta_{2}$-m-independent manner, the overall HLA class I expression score was based on expression of the heavy chains. If either or all the heavy chains were expressed normally, overall HLA class I expression was considered to be normal. Total loss of all heavy chains was counted as total HLA class I loss, and all other cases were counted as partial loss.

As shown in Table 2, expression of all APM components except LMP10, calnexin, and calreticulin was lost in some of the cases. This down-regulation was significantly associated with HLA class I down-regulation for all components, except ERAP1. Moreover, defects in overall TAP expression (defined as partial or total loss of either or both of the TAP subunits) were significantly associated with 
Fig. 1 Immunohistochemical analysis. Staining patterns of a-c HLA-A, d-f HLA-B/-C, g-i LMP7 and $\mathbf{j}-\mathbf{l}$ TAP1 showing a, $\mathbf{d}, \mathbf{g}, \mathbf{j}$ normal expression, $\mathbf{b}, \mathbf{e}, \mathbf{h}, \mathbf{k}$ partial loss of expression and $\mathbf{c}, \mathbf{f}, \mathbf{i}, \mathbf{l}$ total loss of expression
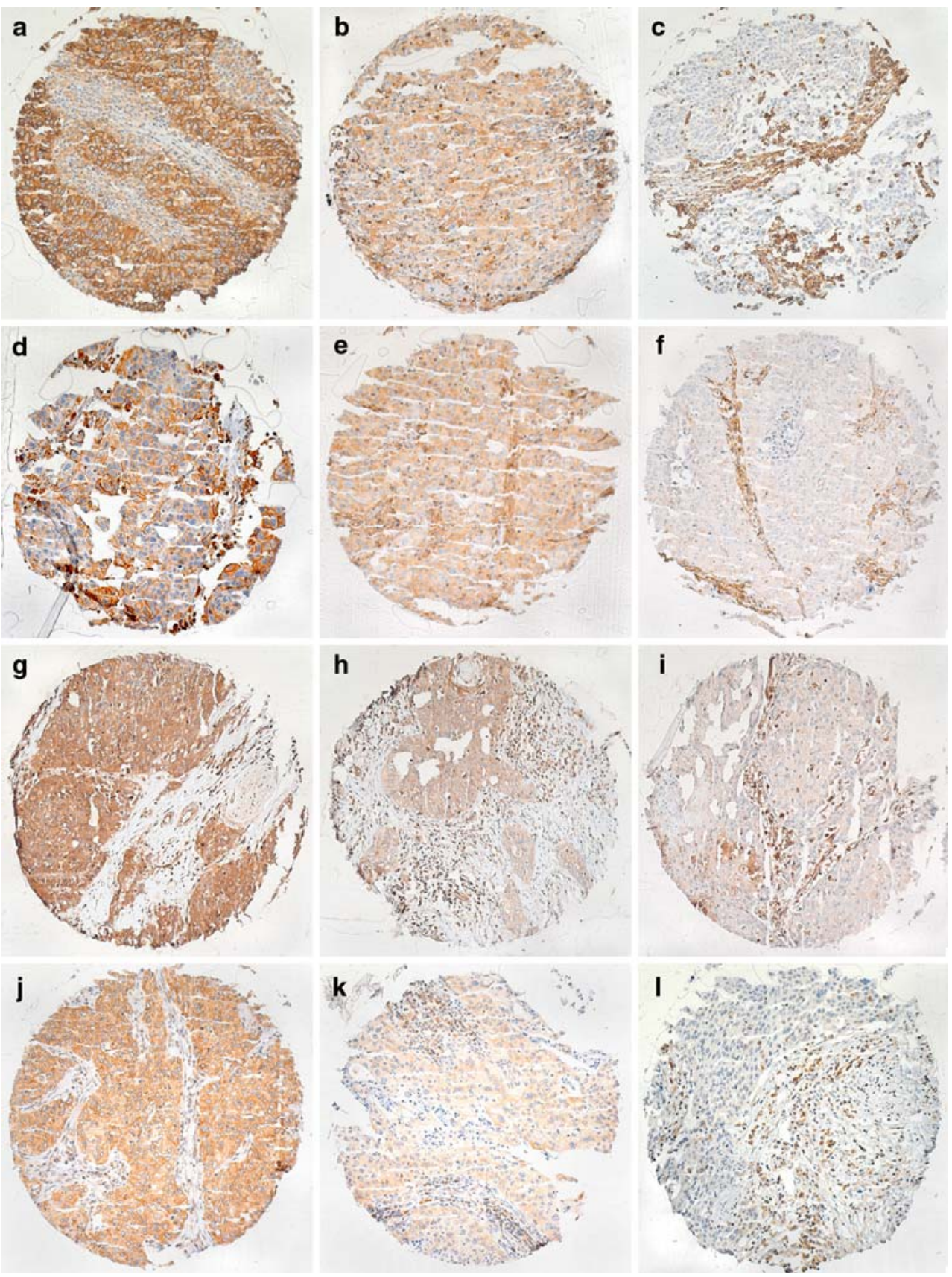

HLA class I down-regulation $(P<0.001)$. Down-regulation of any APM component was significantly associated with HLA class I down-regulation $(P<0.001)$.

\section{Association with histopathological parameters}

Depth of invasion exceeding $15 \mathrm{~mm}$ and vaso-invasive growth are unfavourable prognostic indicators. Of the cases examined, $51(45 \%)$ demonstrated depth of invasion greater than $15 \mathrm{~mm}$ and $38(33 \%)$ demonstrated vasoinvasive growth. Total loss of overall HLA class I expression and of any APM component was found to be significantly associated with depth of invasion exceeding $15 \mathrm{~mm}(P=0.034$ and 0.045 , respectively), while no significant association was found for defective expression of any marker and vaso-invasion.

None of the markers was found to be significantly associated with FIGO stage, corresponding to the lack of a significant association with histopathological parameters. No association of any of the markers with specific HPV status of the patients was detected. Interestingly, partial LMP2 and/or LMP7 loss was significantly associated with an absence of detectable lymph node metastases $(P=0.023$ and 0.021 , respectively). 
Fig. 2 Immunohistochemical staining patterns of ERAP1. a Normal expression. b Partial loss of expression

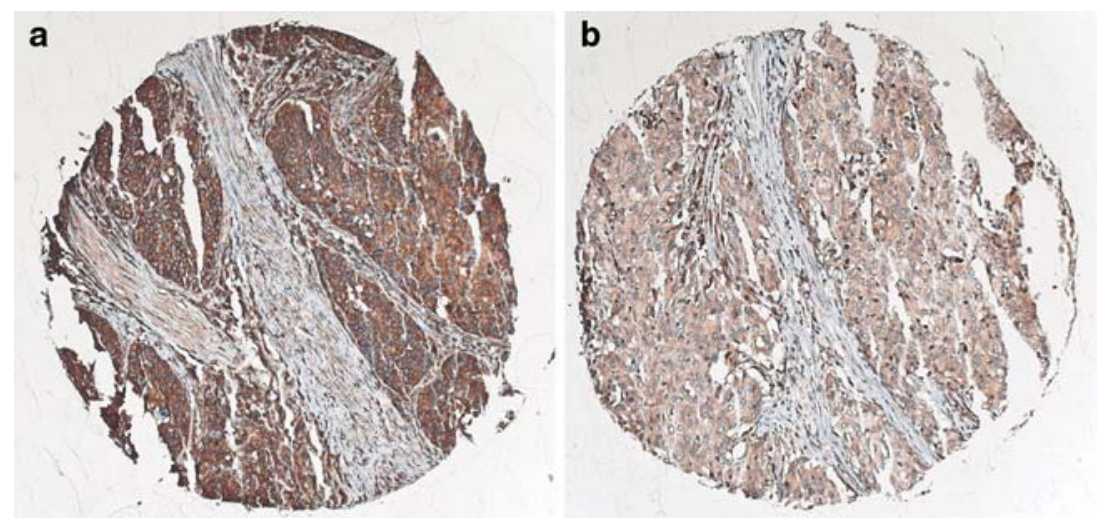

Table 1 Expression of HLA class I heavy chains, $\beta_{2}$-microglobulin and overall HLA class I

\begin{tabular}{|c|c|c|c|c|c|c|c|c|c|c|c|c|}
\hline & \multicolumn{3}{|l|}{ HLA-A } & \multicolumn{3}{|c|}{ HLA-B/-C } & \multicolumn{3}{|c|}{$\beta_{2}$-microglobulin } & \multicolumn{3}{|c|}{ HLA class I } \\
\hline & $\begin{array}{l}\text { Total } \\
\text { loss }\end{array}$ & $\begin{array}{l}\text { Partial } \\
\text { loss }\end{array}$ & $\begin{array}{l}\text { Normal } \\
\text { expression }\end{array}$ & $\begin{array}{l}\text { Total } \\
\text { loss }\end{array}$ & $\begin{array}{l}\text { Partial } \\
\text { loss }\end{array}$ & $\begin{array}{l}\text { Normal } \\
\text { expression }\end{array}$ & $\begin{array}{l}\text { Total } \\
\text { loss }\end{array}$ & $\begin{array}{l}\text { Partial } \\
\text { loss }\end{array}$ & $\begin{array}{l}\text { Normal } \\
\text { expression }\end{array}$ & $\begin{array}{l}\text { Total } \\
\text { loss }\end{array}$ & $\begin{array}{l}\text { Partial } \\
\text { loss }\end{array}$ & $\begin{array}{l}\text { Normal } \\
\text { expression }\end{array}$ \\
\hline Overall $(n=109)$ & $36(33)$ & $22(20)$ & $51(47)$ & $24(22)$ & $29(27)$ & $56(51)$ & $8(7)$ & $28(26)$ & $73(67)$ & $21(19)$ & $24(22)$ & $63(59)$ \\
\hline \multicolumn{13}{|l|}{ Tumour type } \\
\hline $\mathrm{AC}(n=26)$ & $6(23)$ & $5(19)$ & $15(58)$ & $4(15)$ & $8(31)$ & $14(54)$ & $1(4)$ & $7(27)$ & $18(69)$ & $4(15)$ & $5(19)$ & $17(66)$ \\
\hline $\operatorname{SCC}(n=83)$ & $30(36)$ & $17(21)$ & $36(43)$ & $20(24)$ & $21(25)$ & $42(51)$ & $7(8)$ & $21(25)$ & $55(67)$ & $17(21)$ & $19(23)$ & $46(56)$ \\
\hline
\end{tabular}

Data shown as $n(\%)$

Table 2 Overall and histological type-specific expression of APM components

\begin{tabular}{|c|c|c|c|c|c|c|c|c|c|c|c|c|}
\hline & \multicolumn{4}{|c|}{ Overall $(n=101)$} & \multicolumn{4}{|c|}{$\mathrm{AC}(n=25)$} & \multicolumn{4}{|c|}{$\operatorname{SCC}(n=76)$} \\
\hline & $\begin{array}{l}\text { Total } \\
\text { loss }\end{array}$ & $\begin{array}{l}\text { Partial } \\
\text { loss }\end{array}$ & $\begin{array}{l}\text { Normal } \\
\text { expression }\end{array}$ & $P$ & $\begin{array}{l}\text { Total } \\
\text { loss }\end{array}$ & $\begin{array}{l}\text { Partial } \\
\text { loss }\end{array}$ & $\begin{array}{l}\text { Normal } \\
\text { expression }\end{array}$ & $P$ & $\begin{array}{l}\text { Total } \\
\text { loss }\end{array}$ & $\begin{array}{l}\text { Partial } \\
\text { loss }\end{array}$ & $\begin{array}{l}\text { Normal } \\
\text { expression }\end{array}$ & $P$ \\
\hline LMP2 & $14(14)$ & $30(30)$ & $57(56)$ & 0.014 & $5(20)$ & $11(44)$ & $9(36)$ & 0.772 & $9(12)$ & $19(25)$ & $48(63)$ & 0.001 \\
\hline LMP7 & $3(3)$ & $16(16)$ & $82(81)$ & $<0.001$ & $2(8)$ & $9(36)$ & $14(56)$ & 0.100 & $1(1)$ & $7(9)$ & $68(90)$ & $<0.001$ \\
\hline LMP10 & 0 & 0 & $101(100)$ & & & & & & & & & \\
\hline TAP1 & $4(4)$ & 19 (19) & $78(77)$ & $<0.001$ & $0(0)$ & $4(16)$ & $21(84)$ & 0.230 & $4(5)$ & $15(20)$ & $57(75)$ & 0.001 \\
\hline TAP2 & $11(11)$ & $26(26)$ & $64(63)$ & $<0.001$ & $4(16)$ & $6(24)$ & $15(60)$ & 0.005 & $7(9)$ & $20(26)$ & $49(65)$ & $<0.001$ \\
\hline ERAP1 & 0 & $14(15)$ & $80(85)$ & 0.314 & $0(0)$ & $2(9)$ & $20(91)$ & 0.582 & 0 & $12(16)$ & $60(84)$ & 0.307 \\
\hline Tapasin & $1(1)$ & $24(24)$ & $76(75)$ & $<0.001$ & $0(0)$ & $10(40)$ & $15(60)$ & 0.284 & $1(1)$ & $14(18)$ & $61(81)$ & $<0.001$ \\
\hline Calnexin & 0 & 0 & $101(100)$ & & & & & & & & & \\
\hline ERp57 & $12(12)$ & $40(40)$ & $49(48)$ & 0.017 & $1(4)$ & $6(24)$ & $18(72)$ & 0.615 & $11(15)$ & $34(45)$ & $31(40)$ & 0.002 \\
\hline Calreticulin & 0 & 0 & $101(100)$ & & & & & & & & & \\
\hline
\end{tabular}

Data shown as $n(\%) . P$ values are shown for association with overall HLA class I down-regulation

Association with overall and disease-free survival

As shown in Table 3 and Figs. 3 and 4, partial loss of overall HLA class I expression was significantly associated with decreased OS, and exhibited a trend towards a significant association with DFS, whereas total loss was not significantly associated with survival. Moreover, partial HLA-A loss (evaluated by $\mathrm{HCA} 2$ reactivity) was significantly associated with decreased OS and DFS, whereas total loss was not significantly associated with survival. Partial, but not total HLA-B/-C loss (assessed by HC10 reactivity) showed a trend towards significant association with decreased survival.

Decreased OS and DFS was significantly associated with total TAP1 loss, but not with normal expression and partial loss. A similar pattern was observed for partial LMP7 loss, though this was not significantly associated with DFS. ERAP1 loss was significantly associated with 
Table 3 HLA class I and APM component expression and 5-years survival rates

\begin{tabular}{|c|c|c|c|c|c|c|}
\hline & \multicolumn{3}{|l|}{ Overall survival } & \multicolumn{3}{|l|}{ Disease-free survival } \\
\hline & Normal expression & Partial loss & Total loss & Normal expression & Partial loss & Total loss \\
\hline HLA-A & $42(83)$ & $12(55)$ & $29(81)$ & $42(83)$ & $13(60)$ & $28(77)$ \\
\hline HLA-B/-C & $45(81)$ & $19(64)$ & $18(75)$ & $46(82)$ & $19(67)$ & $18(73)$ \\
\hline HLA class I & $52(83)$ & $15(61)$ & $16(77)$ & $52(82)$ & $16(65)$ & $15(75)$ \\
\hline LMP7 & $24(74)$ & $17(87)$ & $1(33)$ & $62(76)$ & $13(80)$ & $2(50)$ \\
\hline TAP1 & $61(78)$ & $8(70)$ & $1(33)$ & $63(81)$ & $13(67)$ & $1(25)$ \\
\hline ERAP1 & $66(82)$ & $5(38)$ & - & $65(81)$ & $7(50)$ & - \\
\hline
\end{tabular}

Data shown as $n(\%)$

decreased OS and DFS as compared to normal ERAP1 expression.

Multivariate analysis

As expected, well known prognostic parameters such as depth of infiltration exceeding $15 \mathrm{~mm}$, presence of vasoinvasive growth, and presence of lymph node metastases were significantly associated with a shorter survival, while FIGO-stages of IB2 or more showed a significant association with shorter DFS (Table 4). HPV type was not significantly associated with survival (data not shown). Subsequently, multivariate analysis was performed on overall HLA class I, TAP1, LMP7 (for OS only) and ERAP1 expression, combined with the aforementioned prognostic factors. ERAP1 down-regulation was demonstrated to be an independent prognostic parameter for shorter OS (hazard ratio (HR) 3.08; 95\% CI 1.07-8.90; $P=0.037$ ) and shorter DFS (HR 2.84; 95\% CI 1.01-8.03; $P=0.049$ ), as was presence of lymph node metastases (HR 7.02; 95\% CI 2.55-19.32; $P<0.001$ for OS and HR 6.64; 95\% CI 2.53-17.44; $P<0.001$ for DFS). Total TAP1 loss was also an independent predictor for shorter DFS (HR 6.72; 95\% CI 1.29-35.06; $P=0.024$ ).

\section{Discussion}

In the present study we have investigated HLA class I and APM component expression in cervical carcinoma patients and have evaluated the relationship of these factors with survival and other clinicopathological parameters.

Our results show that HLA class I down-regulation occurs frequently ( $40 \%$ of cases) in cervical carcinoma, corresponding to previous reports [19, 21]. Synchronous partial or total loss of various APM components was found in a substantial number of cases and was demonstrated to be significantly associated with HLA class I down-regulation. We observed partial HLA class I loss to be significantly associated with decreased OS. Of the APM components, TAP1, LMP7 and ERAP1 down-regulation exhibited significant associations with decreased survival. ERAP1 down-regulation was shown to be an independent, significant predictor of shorter survival.

Though defective APM component expression is hypothesized to be an important mechanism of HLA class I down-regulation and therefore immune evasion by tumour cells, information regarding APM down-regulation in carcinomas is limited. This is partly due to the scarcity of reliable antibodies for most APM components other than the TAP subunits. Furthermore, most previous studies of APM expression in cervical cancer were limited by small sample sizes. Lastly, the use of various scoring methods for evaluation of immunohistochemical staining has rendered proper interpretation of findings difficult, leading to conflicting data, e.g. regarding the frequency of TAP1 loss $[9,18-20]$.

In this study, a panel of recently reported monoclonal antibodies was used to investigate expression of all known APM components in a large set of cervical carcinoma lesions using a TMA-based approach [30-33]. Staining was evaluated according to a previously reported quality control system [37], which is more stringent in defining total and partial expression loss than other evaluation methods, which consider $\leq 25 \%$ positive cells as total loss. The system used here defines total loss as complete or nearcomplete negativity and is therefore more reliable. Moreover, by excluding $\beta_{2}$-m-negative cases from the analysis, all outcome parameters can be reliably correlated to expression levels of the various APM components, as any influence of defective APM expression will only be detectable in a $\beta_{2}$-m-positive setting.

As an APM component, ERAP1 has been shown to be an important determinant of the repertoire of HLA class I-presented peptides [14, 34, 38-41]. However, to date no systematic investigation of its potential role in HLA class I down-regulation in tumours has been performed. To our knowledge, this is the first description of ERAP1 downregulation in a human carcinoma. Additionally, we have identified ERAP1 loss as an independent predictor of 
Fig. 3 Kaplan-Meier curves for overall survival. Patients with normal expression, partial loss, and total loss of a HLA-A, b HLA-B/-C, c overall HLA class I, d LMP7, e TAP1 and f ERAP1 expression. $P$ values are shown for comparison with normal expression
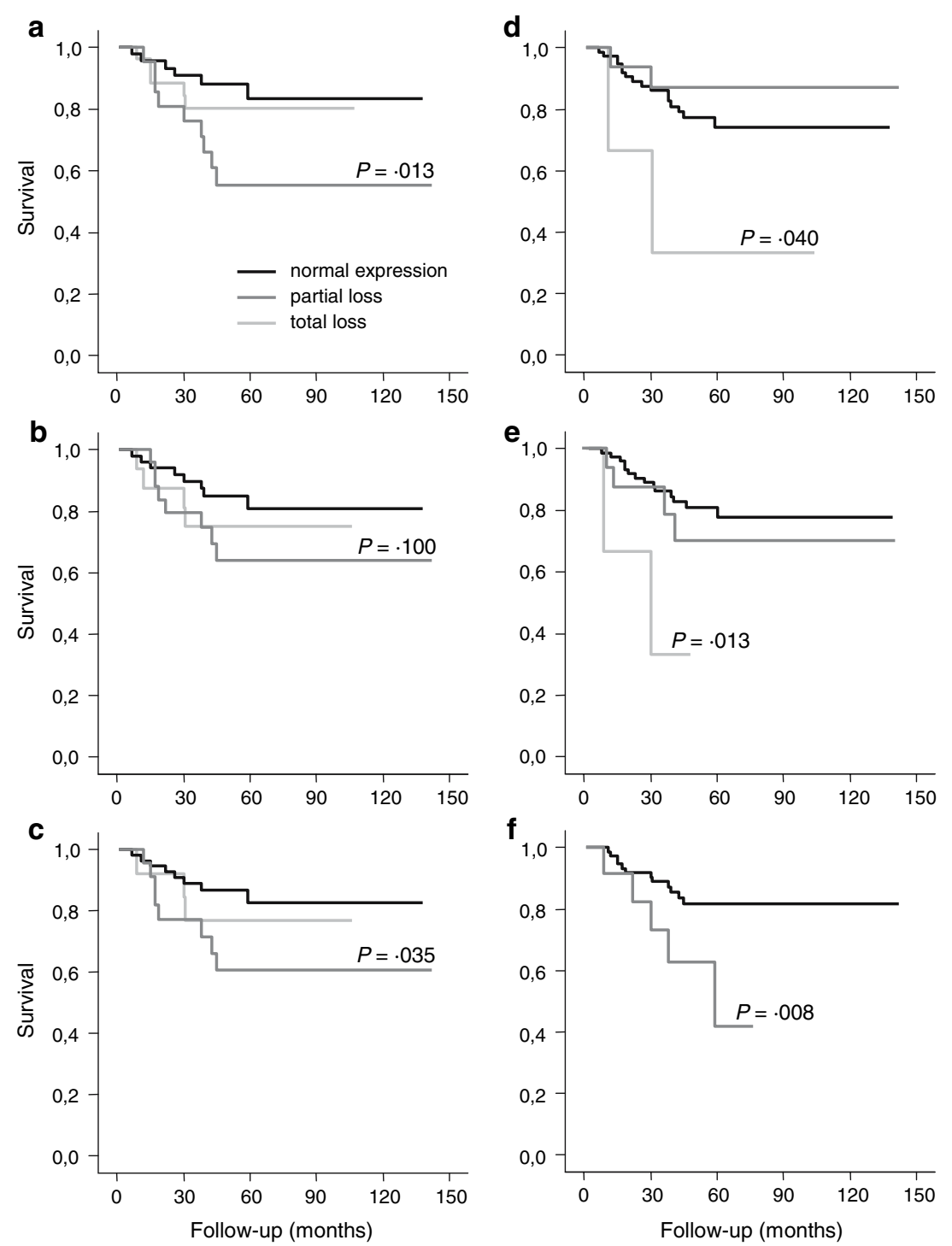

shorter survival, in the absence of associated HLA class I down-regulation. A possible explanation for this observation is the role of ERAP1 in shaping the antigen repertoire: ERAP1 down-regulation may lead to the preferential loading and presentation of non-tumour-associated peptides, thereby yielding a less immunogenic phenotype and facilitating tumour growth and progression. This hypothesis is supported by previous reports on the disrupted nature of presented peptide repertoire in ERAP1-deficient mice [41, 42]. Also, the effect of down-regulation of several APM components on the presentation of specific HPV 16 E6-derived epitopes has been firmly established [43-45].

Although we found that total TAP1 loss was an independent predictor for shorter DFS, this finding should be interpreted with caution as only four patients exhibited this loss. The decreased significance of ERAP1 as an independent predictor for shorter DFS can be explained by the interdependency of ERAP1-mediated peptide trimming and TAP-mediated peptide transport.

An interesting finding in this study is the observed association of LMP2 and LMP7 down-regulation with an absence of lymph node metastasis; this finding is seemingly corroborated by reports that certain immunogenic peptides in melanoma and renal-cell carcinoma are not generated by immunoproteasomes and that presentation of these peptides is reduced upon up-regulation of the immunoproteasomal subunits [46]. Though altered proteasomal cleavage of endogenous proteins can lead to an alternative, less immunogenic antigen repertoire, an adequate mechanism for the association of LMP downregulation with an absence of lymph node metastases remains to be postulated.

Interestingly, we have recently observed that genetic variation in various APM components is significantly 
Fig. 4 Kaplan-Meier curves for disease-free survival. Patients with normal expression, partial loss, and total loss of a HLA-A, b HLA-B/-C, c overall HLA class I, d LMP7, e TAP1 and $\mathbf{f}$ ERAP1 expression. $P$ values are shown for comparison with normal expression
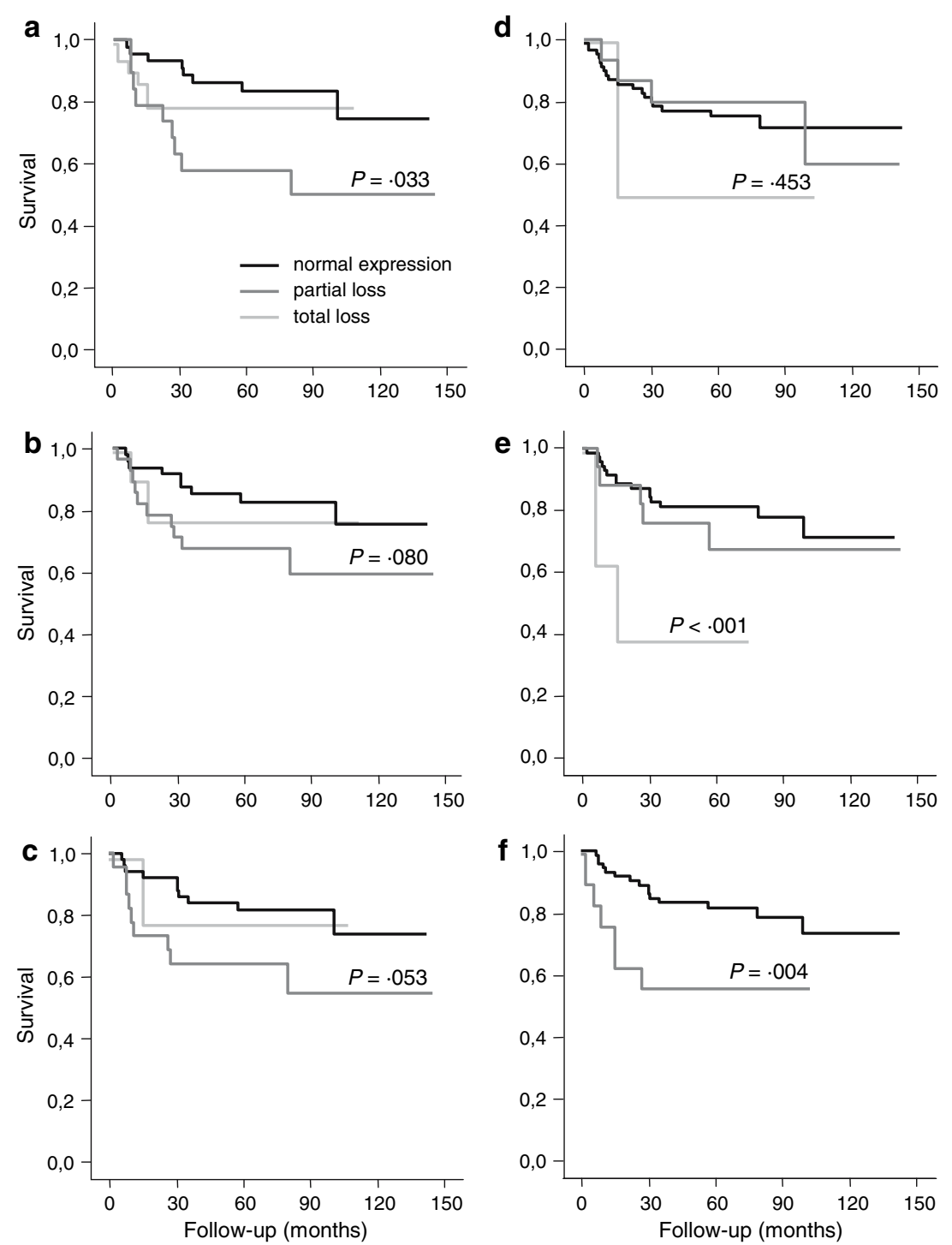

associated with increased cervical carcinoma risk. Although the underlying mechanisms of APM component loss are largely unknown, the occurrence of single nucleotide polymorphisms in the various APM genes could form a genetic basis predisposing to either mutations, or regulatory down-regulation [47, 48].

Partial HLA class I loss was observed to be significantly associated with decreased OS; correspondingly, reports in colon cancer have shown partial, but not total HLA class I loss to be associated with worse OS [49]. This finding can be explained by the ability of low levels of HLA class I molecules to provide tumour cells with an escape not only from cytotoxic $\mathrm{T}$ lymphocyte-mediated, but also from natural killer (NK) cell-mediated cytotoxicity [2]. This hypothesis is further supported by the association of decreased survival with total TAP1 loss, which has been shown previously to lead to partial HLA class I loss $[2,50-52]$. In the present study, an association of loss or down-regulation of expression of the various APM molecules with aberrant HLA expression was observed. Moreover, the partial HLA loss observed is most likely an underestimation of the extent of HLA alterations present as immunohistochemical staining on paraffin sections does not reveal loss of specific HLA alleles [8]. However, there are also other aberrations known to lead to HLA class I alterations and it is plausible to hypothesize that all these mechanisms can coexist in the same tumour sample.

In conclusion, we have shown here that defective expression of HLA class I and of various APM components occurs frequently in cervical carcinoma. Partial HLA class I loss and total TAP1 loss are associated with decreased survival, while down-regulation of immunoproteasomal subunits is associated with decreased risk of lymphogenic metastasis. Moreover, we report the first description of ERAP1 down-regulation in a human carcinoma, and have found that this is an independent prognostic parameter for 
Table 4 Histopathological parameters and 5-years survival rates

\begin{tabular}{|c|c|c|c|c|}
\hline & \multicolumn{2}{|c|}{ Overall survival } & \multicolumn{2}{|c|}{ Disease-free survival } \\
\hline & $\%$ & $P$ & $\%$ & $P$ \\
\hline \multicolumn{5}{|l|}{ Depth of infiltration } \\
\hline$<15 \mathrm{~mm}(n=59)$ & 88 & & 90 & \\
\hline$\geq 15 \mathrm{~mm}(n=51)$ & 60 & $<0.001$ & 57 & $<0.001$ \\
\hline \multicolumn{5}{|l|}{ Vaso-invasion } \\
\hline Absent $(n=68)$ & 81 & & 85 & \\
\hline Present $(n=38)$ & 59 & 0.031 & 54 & 0.002 \\
\hline \multicolumn{5}{|l|}{ Lymph nodes } \\
\hline Negative $(n=80)$ & 86 & & 86 & \\
\hline Positive $(n=27)$ & 43 & $<0.001$ & 39 & $<0.001$ \\
\hline \multicolumn{5}{|c|}{ Histopathological class } \\
\hline $\operatorname{SCC}(n=84)$ & 75 & & 76 & \\
\hline $\mathrm{AC}(n=26)$ & 77 & 0.924 & 74 & 0.662 \\
\hline \multicolumn{5}{|l|}{ FIGO stage } \\
\hline$\leq \mathrm{IB} 1(n=52)$ & 82 & & 91 & \\
\hline$\geq \operatorname{IB} 2(n=53)$ & 69 & 0.060 & 57 & 0.002 \\
\hline
\end{tabular}

decreased survival. A complete understanding of the mechanisms and relevance of HLA class I and APM component down-regulation and immune evasion may contribute to the rational design of tumour vaccines and T-cell-based immunotherapies.

Acknowledgements The authors thank Dr. M. Tsujimoto, RIKEN, Wako, Saitama, Japan for kindly providing the anti-ERAP1 antibody.

\section{References}

1. Tindle RW (2002) Immune evasion in human papillomavirusassociated cervical cancer. Nat Rev Cancer 2:59-65

2. Garrido F, Ruiz-Cabello F, Cabrera T, Perez-Villar J, LopezBotet M, Duggan-Keen M, Stern P (1997) Implications for immunosurveillance of altered HLA class I phenotypes in human tumours. Immunol Today 18:89-95

3. Ruiz-Cabello F, Cabrera T, Lopez-Nevot M, Garrido F (2002) Impaired surface antigen presentation in tumours: implications for T cell-based immunotherapy. Semin Cancer Biol 12:15-24

4. Seliger B, Maeurer MJ, Ferrone S (2000) Antigen-processing machinery breakdown and tumour growth. Immunol Today 21:455-464

5. Seliger B, Cabrera T, Garrido F, Ferrone S (2002) HLA class I antigen abnormalities and immune escape by malignant cells. Semin Cancer Biol 12:3-13

6. Bjorkman PJ, Parham P (1990) Structure, function, and diversity of class I major histocompatibility complex molecules. Annu Rev Biochem 59:253-288

7. Natarajan K, Li H, Mariuzza RA, Margulies DH (1999) MHC class I molecules, structure and function. Rev Immunogenet $1: 32-46$

8. Koopman LA, van der Slik AR, Giphart MJ, Fleuren GJ (1999) Human leukocyte antigen class I gene mutations in cervical cancer. J Natl Cancer Inst 91:1669-1677
9. Brady CS, Bartholomew JS, Burt DJ, Duggan-Keen MF, Glenville S, Telford N, Little AM, Davidson JA, Jimenez P, RuizCabello F, Garrido F, Stern P (2000) Multiple mechanisms underlie HLA dysregulation in cervical cancer. Tissue Antigens 55:401-411

10. Koopman LA, Corver W, van der Slik AR, Giphart MJ, Fleuren GJ (2000) Multiple genetic alterations cause frequent and heterogeneous human histocompatibility leukocyte antigen class I loss in cervical cancer. J Exp Med 191:961-976

11. York IA, Rock KL (1996) Antigen processing and presentation by the class I major histocompatibility complex. Annu Rev Immunol 14:369-396

12. Gromme M, Neefjes J (2002) Antigen degradation or presentation by MHC class I molecules via classical and non-classical pathways. Mol Immunol 39:181-202

13. Van Kaer L (2002) Major histocompatibility complex class I-restricted antigen processing and presentation. Tissue Antigens 60:1-9

14. York IA, Chang SC, Saric T, Keys JA, Favreau JM, Goldberg AL, Rock K (2002) The ER aminopeptidase ERAP1 enhances or limits antigen presentation by trimming epitopes to 8-9 residues. Nat Immunol 3:1177-1184

15. Meissner M, Reichert TE, Kunkel M, Gooding W, Whiteside TL, Ferrone S, Seliger B (2005) Defects in the human leukocyte antigen class I Antigen Processing Machinery in head and neck squamous cell carcinoma: association with clinical outcome. Clin Cancer Res 11:2552-2560

16. Facoetti A, Nano R, Zelini P, Morbini P, Benericetti E, Ceroni M, Campoli M, Ferrone S (2005) Human leukocyte antigen and antigen processing machinery component defects in astrocytic tumours. Clin Cancer Res 11:8304-8311

17. Seliger B, Ritz U, Ferrone S (2006) Molecular mechanisms of HLA class I antigen abnormalities following viral infection and transformation. Int J Cancer 118:129-138

18. Ritz U, Momburg F, Pilch H, Huber C, Maeurer MJ, Seliger B (2001) Deficient expression of components of the MHC class I antigen processing machinery in human cervical carcinoma. Int J Oncol 19:1211-1220

19. Cromme FV, Airey J, Heemels MT, Ploegh HL, Keating PJ, Stern PL, Meijer CJ, Walboomers JM (1994) Loss of transporter protein, encoded by the TAP-1 gene, is highly correlated with loss of HLA expression in cervical carcinomas. J Exp Med 179:335-340

20. Keating PJ, Cromme FV, Duggan-Keen M, Snijders PJ, Walboomers JM, Hunter RD, Dyer PA, Stern PL (1995) Frequency of down-regulation of individual HLA-A and -B alleles in cervical carcinomas in relation to TAP-1 expression. Br J Cancer 72:405411

21. Cromme FV, van Bommel PF, Walboomers JM, Gallee MP, Stern PL, Kenemans P, Helmerhorst TJ, Stukart MJ, Meijer CJ (1994) Differences in MHC and TAP-1 expression in cervical cancer lymph node metastases as compared with the primary tumours. Br J Cancer 69:1176-1181

22. Hilders CG, Houbiers JG, Krul EJ, Fleuren GJ (1994) The expression of histocompatibility-related leukocyte antigens in the pathway to cervical carcinoma. Am J Clin Pathol 101:5-12

23. Hilders CG, Munoz IM, Nooyen Y, Fleuren GJ (1995) Altered HLA expression by metastatic cervical carcinoma cells as a factor in impaired immune surveillance. Gynecol Oncol 57:366-375

24. van Driel WJ, Tjiong MY, Hilders CG, Trimbos BJ, Fleuren GJ (1996) Association of allele-specific HLA expression and histopathologic progression of cervical carcinoma. Gynecol Oncol 62:33-41

25. Torhorst J, Bucher C, Kononen J, Haas P, Zuber M, Kochli OR, Mross F, Dieterich H, Moch H, Mihatsch M, Kallioniemi OP, Sauter G (2001) Tissue microarrays for rapid linking of molecular changes to clinical endpoints. Am J Pathol 159:2249-2256 
26. Ruiz C, Seibt S, Kuraya KA, Siraj AK, Mirlacher M, Schraml P, Maurer R, Spichtin H, Torhorst J, Popovska S, Simon R, Souter G (2005) Tissue microarrays for comparing molecular features with proliferation activity in breast cancer. Int J Cancer 118:2190-2194

27. Stam NJ, Spits H, Ploegh HL (1986) Monoclonal antibodies raised against denatured HLA-B locus heavy chains permit biochemical characterization of certain HLA-C locus products. J Immunol 137:2299-2306

28. Sernee MF, Ploegh HL, Schust DJ (1998) Why certain antibodies cross-react with HLA-A and HLA-G: epitope mapping of two common MHC class I reagents. Mol Immunol 35:177-188

29. Perosa F, Luccarelli G, Prete M, Favoino E, Ferrone S, Dammacco F (2003) Beta 2-microglobulin-free HLA class I heavy chain epitope mimicry by monoclonal antibody HC-10-specific peptide. J Immunol 171:1918-1926

30. Bandoh N, Ogino T, Cho HS, Hur SY, Shen J, Wang X, Kato S, Miyokawa N, Harabuchi Y, Ferrone S (2005) Development and characterization of human constitutive proteasome and immunoproteasome subunit-specific monoclonal antibodies. Tissue Antigens 66:185-194

31. Wang X, Campoli M, Cho HS, Ogino T, Bandoh N, Shen J, Hur SY, Kageshita T, Ferrone S (2005) A method to generate antigen-specific $\mathrm{mAb}$ capable of staining formalin-fixed, paraffinembedded tissue sections. J Immunol Methods 299:139-151

32. Ogino T, Wang X, Kato S, Miyokawa N, Harabuchi Y, Ferrone S (2003) Endoplasmic reticulum chaperone-specific monoclonal antibodies for flow cytometry and immunohistochemical staining. Tissue Antigens 62:385-393

33. Hattori A, Kitatani K, Matsumoto H, Miyazawa S, Rogi T, Tsuruoka N, Mizutani S, Natori Y, Tsujimoto M (2000) Characterization of recombinant human adipocyte-derived leucine aminopeptidase expressed in Chinese hamster ovary cells. J Biochem 128:755-762

34. Saric T, Chang S, Hattori A, York IA, Markant S, Rock KL, Tsujimoto M, Goldberg AL (2002) An IFN-gamma-induced aminopeptidase in the ER, ERAP1, trims precursors to MHC class I-presented peptides. Nat Immunol 3:1169-1176

35. Shibata D, Ando H, Iwase A, Nagasaka T, Hattori A, Tsujimoto M, Mizutani S (2004) Distribution of adipocyte-derived leucine aminopeptidase (A-LAP)/ER-aminopeptidase (ERAP)-1 in human uterine endometrium. J Histochem Cytochem 52:1169-1175

36. Hazelbag S, Gorter A, Kenter GG, van den Broek L, Fleuren GJ (2002) Transforming growth factor-betal induces tumour stroma and reduces tumour infiltrate in cervical cancer. Hum Pathol 33:1193-1199

37. Ruiter DJ, Ferrier CM, van Muijen GN, Henzen-Logmans S, Kennedy S, Kramer MD, Nielsen B, Schmitt M (1998) Quality control of immunohistochemical evaluation of tumour-associated plasminogen activators and related components. European BIOMED-1 Concerted Action on Clinical Relevance of Proteases in Tumour Invasion and Metastasis. Eur J Cancer 34:1334-1340

38. Falk K, Rotzschke O (2002) The final cut: how ERAP1 trims MHC ligands to size. Nat Immunol 3:1121-1122

39. Chang SC, Momburg F, Bhutani N, Goldberg AL (2005) The ER aminopeptidase, ERAP1, trims precursors to lengths of MHC class I peptides by a "molecular ruler" mechanism. Proc Natl Acad Sci USA 102:17107-17112
40. Saveanu L, Carroll O, Lindo V, Del Val M, Lopez D, Lepelletier Y, Greer F, Schomburg L, Fruci D, Niedermann G, van Endert PM (2005) Concerted peptide trimming by human ERAP1 and ERAP2 aminopeptidase complexes in the endoplasmic reticulum. Nat Immunol 6:689-697

41. Hammer GE, Gonzalez F, Champsaur M, Cado D, Shastri N (2006) The aminopeptidase ERAAP shapes the peptide repertoire displayed by major histocompatibility complex class I molecules. Nat Immunol 7:103-112

42. Yan J, Parekh VV, Mendez-Fernandez Y, Olivares-Villagomez D, Dragovic S, Hill T, Roopenian DC, Joyce S, Van Kaer L (2006) In vivo role of ER-associated peptidase activity in tailoring peptides for presentation by MHC class Ia and class Ib molecules. J Exp Med 203:647-659

43. Evans M, Borysiewicz LK, Evans AS, Rowe M, Jones M, Gileadi U, Cerundolo V, Man S (2001) Antigen processing defects in cervical carcinomas limit the presentation of a CTL epitope from human papillomavirus 16 E6. J Immunol 167:5420-5428

44. Barnden MJ, Purcell AW, Gorman J, McCluskey J (2000) Tapasin-mediated retention and optimization of peptide ligands during the assembly of class I molecules. J Immunol 165:322330

45. Garbi N, Tan P, Diehl AD, Chambers BJ, Ljunggren H, Momburg F, Hammerling GJ (2000) Impaired immune responses and altered peptide repertoire in tapasin-deficient mice. Nat Immunol $1: 234-238$

46. Morel S, Levy F, Burlet-Schiltz O, Brasseur F, Probst-Kepper M, Peitrequin AL, Monsarrat B, Van Velthoven R, Cerottini JC, Boon T, Gairin JE, Van den Eynde BJ (2000) Processing of some antigens by the standard proteasome but not by the immunoproteasome results in poor presentation by dendritic cells. Immunity 12:107-117

47. Gostout BS, Poland GA, Calhoun ES, Sohni Y, Giuntoli RL, McGovern RM, Sloan J, Cha S, Persing DH (2003) TAP1, TAP2, and HLA-DR2 alleles are predictors of cervical cancer risk. Gynecol Oncol 88:326-332

48. Cao B, Tian X, Li Y, Jiang P, Ning T, Xing H, Zhao Y, Zhang C, Shi X, Chen D, Shen Y, Ke Y (2005) LMP7/TAP2 gene polymorphisms and HPV infection in esophageal carcinoma patients from a high incidence area in China. Carcinogenesis 26:12801284

49. Watson NF, Ramage JM, Madjd Z, Spendlove I, Ellis IO, Scholefield JH, Durrant LG (2006) Immunosurveillance is active in colorectal cancer as downregulation but not complete loss of MHC class I expression correlates with a poor prognosis. Int $\mathbf{J}$ Cancer 118:6-10

50. Seliger B, Maeurer MJ, Ferrone S (1997) TAP off-tumours on. Immunol Today 18:292-299

51. Young NT, Mulder A, Cerundolo V, Claas FH, Welsh KI (1998) Expression of HLA class I antigens in transporter associated with antigen processing (TAP)-deficient mutant cell lines. Tissue Antigens 52:368-373

52. Kageshita T, Hirai S, Ono T, Hicklin DJ, Ferrone S (1999) Down-regulation of HLA class I antigen-processing molecules in malignant melanoma: association with disease progression. Am J Pathol 154:745-754 